\title{
Teaching Digital Image Processing Topics via Matlab Techniques
}

\author{
Ali Abdullah Yahya
}

\begin{abstract}
Utilizing visual aids in teaching have become extremely important in the modern age of information technology. Most of the conventional teaching techniques of image processing are focused on traditional means; teachers, textbooks and classrooms. These techniques are stood as a stumbling block in front of image technology development. Matlab can be considered as a matrix-oriented computing engine. Since image can be thought as a matrix, therefore, Matlab is the ideal program for image processing. This work proposes to deepen the perception the topics of digital image processing by utilizing Matlab. We believe that teaching the digital image processing with Matlab will lead to moving the enthusiasm of students and helping them to think creatively. Teaching digital image processing topics with Matlab will make these topics easier, that is due to using visual, interactive as well as experimental methods will attract students' attention more than traditional methods, which will lead to building deeper understanding and better memory for digital image processing topics.
\end{abstract}

Index Terms-Digital image processing, Matlab techniques, conventional teaching techniques.

\section{INTRODUCTION}

As we know image processing is considered as the backbone of the emerging visual communication. For that reason, this field has become very interesting for the vast majority of students. Nevertheless, this field actually need student who has a wide imagination and creative thinking to understand the topics of image processing.

The most of conventional image processing teaching methods ignore the mutual relationship between image processing curriculum and other related curricula. Without taking this relationship into consideration students will be unable to get a clear understanding of the teaching system [1]-[5].

Curriculum materials in mathematics and science for the upper elementary and secondary levels have been developed by Greenberg et al. [4]. This study has shown that image processing is an efficient and enjoyable way to study the application of science and mathematics to the real world. In addition to that, the authors emphasized that using image processing is a successful means with which to involve students in inquiry and discovery learning.

Digital image processing subjects involve; image segmentation, image restoration, image enhancement, image transformation, image coding and so on [6]. Consequently, teaching these subjects will be more complicated and abstract

Manuscript received April 12, 2019; revised July 13, 2019.

A. A. Yahya is with the School of Computer and Information, Anqing Normal University, Anqing, China (e-mail: aselwey1@ hotmail.com). unless we use more effective methods like Maltlab.

To name a few, digital image processing curriculum includes some intricate transformation methods such as Fourier transform frequency domain and transform time domain. From our perspective, explaining these transformation methods with Matlab assistance will lead to eliminating demystification and complexity of these methods.

The main aim of teaching digital image processing is to enable students to understand and use the basic theory knowledge of digital image processing. In addition to making students realize the techniques of digital image processing such as Discrete Cosine Transformation (DCT), Fourier transform, filter design, discrete Fourier transform (DFT) and its implementation using the fast Fourier transform [7].

In our school of computer science and information, digital image processing is considered as a compulsory course. Looking for a suitable teaching method remains a huge challenge for the vast majority of the teachers in our school.

Digital image processing curriculum has a wide background. Familiarity with this background is not an easy task. Traditional teaching methods are no longer effective to face such a challenge. Most of the traditional teaching methods are focusing on the theories and ignoring the importance of utilizing the Matlab as a computing platform for improving and testing a number of applications of digital image processing. Consequently, teaching reform of digital image processing course has become an urgent necessity [8].

As a platform for teaching digital image processing curriculum, Matlab is an ideal platform. Most of the undergraduate students are prefer using $\mathrm{C}++$ in the experimental teaching of image processing, however, it is so difficult applying $\mathrm{C}++$ to digital image processing algorithms. The difficulty of applying $\mathrm{C}++$ will in turn lead to increasing the difficulty degree of learning this course. For that reason, Matlab software should be adopted in the experimental teaching of digital image processing instead of $\mathrm{C}++$.

The vast majority of the conventional teaching methods of digital image processing are base on teachers' explanation. Those methods don't get approbation of students.

Francisco and Campos [3] described a set of simple Graphical User Interfaces (GUIs). GUIs have been developed in Scilab, where the authors have utilized the Scilab image and video processing toolbox package. In this work, the authors tried to apply these interfaces to assist the learning of image processing concepts, in addition to promote students' interest.

In order to develop the traditional teaching model of digital image processing, Wang and Guo [1] proposed some important reforms in the digital image processing curriculum, which comprises teaching objective, content setting, 
experiment platform, and innovative training.

Leavline and Singh [9], proposed a new teaching method for learning the concepts of image processing using Matlab. The authors have developed this method for undergraduate level students with engineering background. According to this study, the proposed method can be adopted in teaching the concepts of image compression, registration, feature extraction, and retrieval.

In this paper, we propose to improve the teaching materials of digital image processing course by utilizing Matlab, which is can be considered as a computing platform that is appropriate for improving the teaching methods of digital image processing and its applications. Teaching the digital image processing topics with Matlab will help students to be more positive and enthusiastic at the class, in addition to engaging them in the learning process smoothly.

The remainder of this paper is organized as follows: Teaching digital image processing topics with Matlab is described in Section II. Some concluding remarks are given in Section III.

\section{Teaching Digital Image Processing Topics With MATLAB}

Matlab can be considered as a high-performance language for visualization graphics tools. It is an ideal language for; graphics, image processing, signal processing and simulation integrated. Utilizing Matlab with the image processing toolbox will help students to make digital image processing topics easy to grasp, in addition to making students learning through the creation of a theoretical understanding based on an interactive instance [10]-[12].

Our proposed teaching system aims to develop digital image processing teaching methods with Matlab. In the proposed system, to increase student's innovation, experiments with Matlab are designed to be from superficial to profound and from simple experiments to innovation experiments. In the proposed technique, experiments involve morphological operations, histogram equalization, discrete Fourier transform, discrete cosine transformation, image denoising filters and image edge detection. Utilizing Matlab for teaching digital image processing will help students to comprehend the design ideas and at the same time enhance students' capacity of analyzing and solving practical problems. Therefore, it will be better if we can concurrently do basic demonstration type experiments synchronized with theory teaching.

By the experimental process with Matlab, we suppose that students will be able to; accomplish virtual simulation about the experiments content, utilize Matlab simulation technology to test the system performance as well as create noise removal algorithms and employ Matlab simulation technology in the experiments notably in the sophisticated experiments.

\section{A. Morphological Operations}

Erosion, dilation, opening and closing, and top-hat and bottom-hat transforms are known as the morphological operations. Erosion and dilation are considered as the basic operations of morphology, while other operations are built from a mixture of these two operations.

\section{1) Erosion}

In erosion, the minimum value of all pixels in the input pixel's neighborhood is considered as the output pixel value, where the output pixel will be set to zero if any one of the pixels was set to zero.

\section{2) Dilation}

In dilation, the maximum value of all the pixels in the input pixel's neighborhood is considered as the output pixel value, where the output pixel will be assigned to one if any of the pixels was assigned to one.

\section{3) Opening and closing}

Opening and closing are the combinations of the fundamental operations of erosion and dilation. In opening, erosion is followed by the dilation, while in closing; dilation is followed by the erosion. Opening is particularly useful for; removing small objects from an image and preserving the shape and size of larger objects in this image.

Opening and closing operations are very helpful for removing artifacts present especially after segmenting the image.

Fig. 1 displays the results of applying erosion and dilation operations to text image, whereas Fig. 2 exposes the results of applying opening and closing operations to circles image.

The aim that expects to be achieved from teaching the morphological operations with Matlab is to enable student to realize the effect of morphological operations on binary images by using structuring element, as well as enable them to grasp the effect of morphological functions on changing the images by applying these functions.

\section{4) Top/bottom hat transformation}

The expected goal of teaching top and bottom hats transformation (TBHT) with Matlab is to realize the real significance of applying TBHT to the image. The main purpose of applying the TBHT is to lighten objects on a dark background and darken objects on a light background, respectively [13]. Consequently, the contrast of the image will be improved.

Fig. 9 shows the results of applying top hat and bottom hat transformation to the rice image.

\section{B. Histogram Equalization}

Histogram equalization is a technique usually used for adjusting the intensities of the image to enhance image contrast, thus we can get an image that is more suitable than the original one.

The histogram of the image is a graph that normally provides information about the number of image's pixels at each different intensity value.

The 8-bit grayscale image has 256 various intensities, and therefore histogram graphically exhibit 256 numbers indicating the pixels distribution amidst the values of the grayscale.

The histogram work theory is summarized in scanning the image in a single path, which pixels that found at each intensity value is kept, whereupon suitable histogram will be built.

The purpose of teaching histogram equalization with 
Matlab is to allow students to realize the relationship between the intensities and the histogram of the image. As well as showing how histogram equalization can improve image quality, in other words, how the simple concept like histogram equalization can greatly affect the quality of the image.

Fig. 3 shows the original image of the boy pout image and its enhanced image. Fig. 4 shows the histograms of the original image of boy pout image and that of the enhanced image.

From the histogram of the original image of Fig. 4, students can remark that nearly all of the intensities of the pixels are centered between 75 and 150, in other words most of the pixels are clustered together in the center of the histogram, which means that the dynamic range of the original image is small, therefore, student can expect that the image has poor contrast. In return, the intensities of the pixels in the histogram of the enhanced image are distributed between 0 and 250, which means that the dynamic range of the enhanced image is large.

From histogram figure, students can also observe that applying the histogram equalization helps them to obtain a better-contrasted image that because histogram equalization plays a good role in distributing the pixels throughout all the range, instead of clustered these pixels around the center of the histogram.

\section{Discrete Fourier and Discrete Cosine Transforms}

The vast majority of students frequently face great difficulty in learning discrete Fourier transform (DFT) and discrete cosine transform (DCT), that because DFT and DCT are highly abstract, as well as the similarity between DFT, DCT and other transforms.

Teaching the concepts of DFT and DCT via Matlab techniques will guide students to realize how to grasp the basic knowledge of DFT and DCT and their implementations, in addition to providing them with a perception of the harmonic content of the image.

Based on utilizing Matlab in teaching the concepts of DFT, students can rigorously discover that DFT represents a discrete-time sequence as a series of coefficients; in addition, it is reversible transformation.

Lena image and its DFT are shown in Fig. 5, while cameraman image and its DCT are shown in Fig. 10.

\section{Image Denoising Filters}

\section{1) Removing salt and pepper, gaussian and speckle noises}

Salt and pepper noise which sometime called impulse noise appears as random white and black pixels over the image [14]. Poisson noise can be defined as a type of noise which can be modeled by a Poisson process. Speckle noise is random values multiplied by pixel values. The most typical filter for removing these noises is median filter. Median filter is the example of a non-linear spatial filter. The basic idea of median filter is to substitute each pixel's value by the median of the gray levels in a neighborhood of that pixel.

Fig. 6,11 and 12 demonstrate the effects of median filter on salt and pepper noise Poisson noise and speckle noise. From Fig. 6, 11 and 12, students can notice the effect of a median filter on an impulsive noise corrupted image. Students can also observe how a simple filter can positively affect on the quality of the denoised image.

Teaching the non-linear filter with Matlab gives students a clear perception of how the impulse noise can be eliminated by applying the median filter.

Table I compares the PSNR results of different images that corrupted by Poisson noise, salt and pepper noise, and speckle noise.

For PSNR we use the following formula:

$$
P S N R=10 \log _{10} \frac{255^{2} \times N_{x} \times N_{y}}{\sum_{i=1}^{N_{x}} \sum_{j=1}^{N_{y}}\left[I_{D}(i, j)-I_{O}(i, j)\right]^{2}}
$$

where $N_{x}$ and $N_{y}$ are the numbers of pixels horizontally and vertically, respectively, and $I_{D}(i, j), I_{O}(i, j)$ are the denoised frame and original frame, respectively.

From Table I, students can observe that increasing the amount of noise is offset by decreasing the PSNR values, which means that the denoising effect of the median filter is worse with increased noise.

2) Removing gaussian noise

Gaussian noise is a kind of white noise produced by random fluctuations in the signal.

Gaussian noise could be seen and heard when we tune TV to a channel.

The ideal filter for teaching how to remove Gaussian noise is average filter.

During teaching Gaussian noise with mean zero, it is expected that students can realize that average filter averages the noise to zero.

From applying the average filter to remove Gaussian noise, we expect that students will understand how is the filter kernel size can affect the noise removal results.

Fig. 7 shows results of applying average filter on Barbara image to remove Gaussian noise, which the average filters are $3 \times 3$ and $7 \times 7$ respectively. From this figure students can observe that $3 \times 3$ average filter left most of the noise without removal, while the output image of applying $7 \times 7$ average filter seems much blurred as compare whit the result of applying $3 \times 3$ average filter.

From teaching image denosing filters with Matlab students can also realize how image can be affected with filtering the low and high frequencies.

\section{E. Edge Detection}

Edge can be defined as a line of pixels appearing in noticeable variations.

Most significant image details are located in the edges. In our daily life there are many different types of edge applications, to name a few; measuring objects size in an image, separating particular objects from their background, identifying and classifying objects.

The most known edge detectors are; Sobel operator which is called row edge detector, Prewitt operator which is called 
column edge detector, Robert's cross operator which is the 2-D spatial gradient measurement of an image and Laplacian of Gaussian edge operator. Laplacian operator can be obtained by considering the difference of differences.

Fig. 8 shows the result of: Laplacian edge detector, Prewitt edge detector, Roberts edge detector and Sobel edge detector respectively. Fig. 13 shows the result of watershed [15].
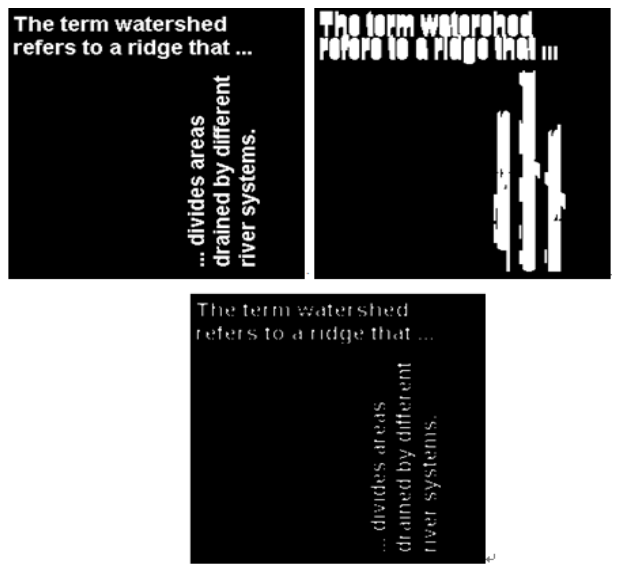

Fig. 1. From left-to right-hand side and from top to bottom, original image, dilated image and eroded image.
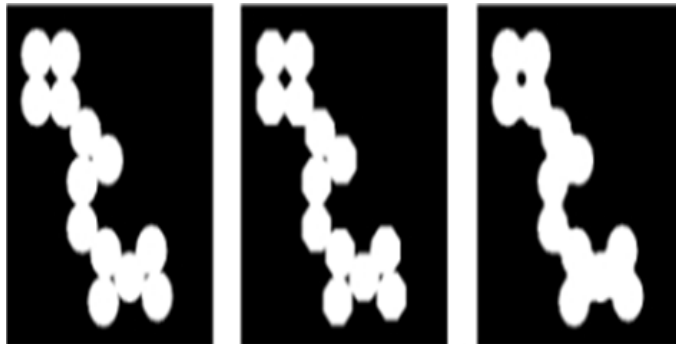

Fig. 2. From left-to right-hand side: original image, opened image and closed image.
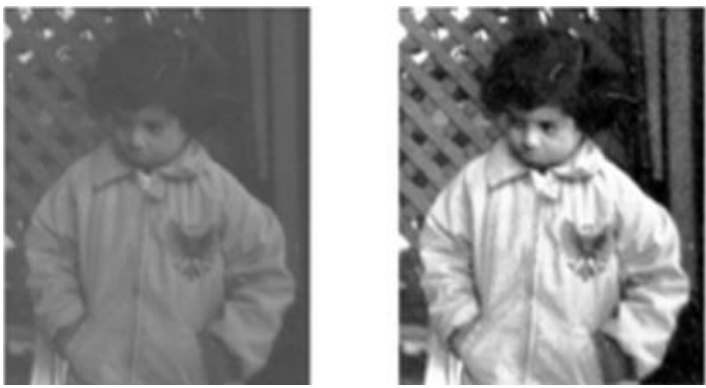

Fig. 3. From left-to right-hand side: original image and enhanced image (threshold at 120).

Segmentation by morphological watersheds embodies many of the concepts of edge detection, thresholding, and region growing.
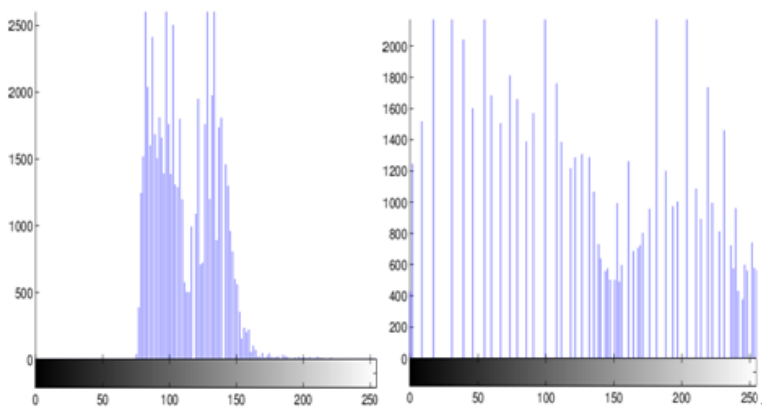

Fig. 4. From left-to right-hand side: histogram of original image in Fig. (threshold at 150) and histogram of enhanced image in Fig. 3 (threshold at $150)$.
Through the use of Matlab in teaching watershed, students can realize that Watershed produces more stable segmentation results, as well as providing simple framework.

From Fig. 8 students can discover that the result of Laplacian mask is the best among the results of the four masks. This mask can detect edges in all directions. In contrast, Prewitt and Sobel masks are better than Roberts mask in terms of detecting diagonal edges.
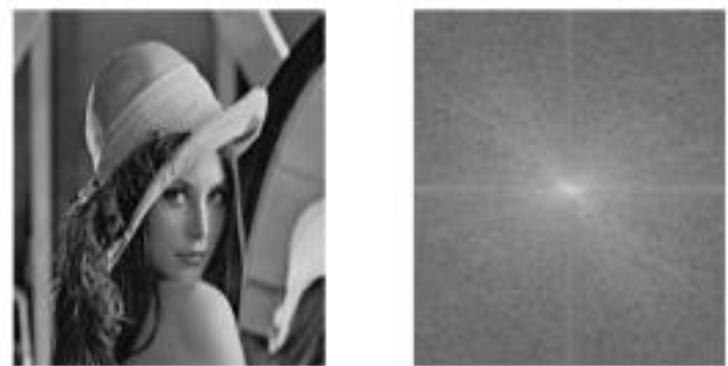

Fig. 5. From left-to right-hand side: original image and its DFT
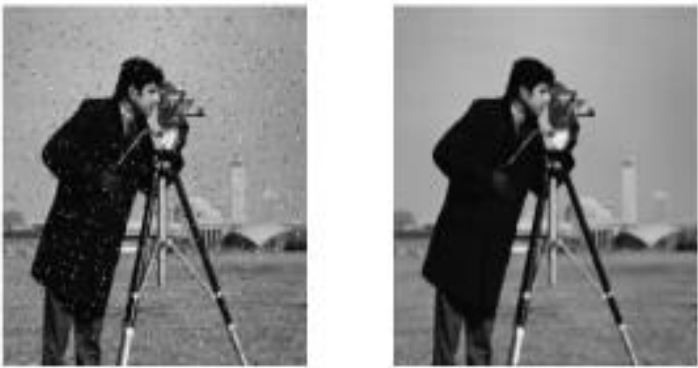

Fig. 6. From left-to right-hand side: noisy image (salt and pepper noise (variance $\sigma^{2}=0.02$ )) and filtered image.
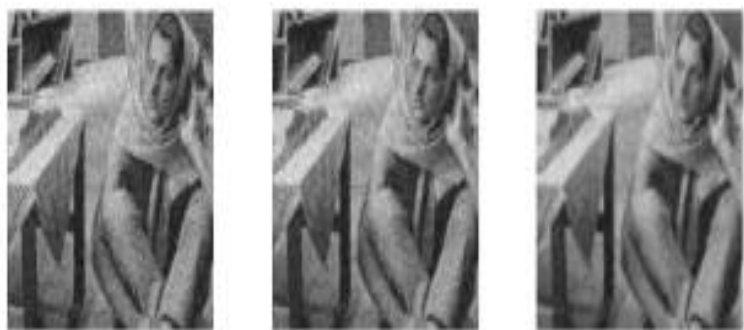

Fig. 7. From left-to right-hand side: noisy image (standard deviation $(\sigma=50))$, filtered image $(3 \times 3$ filtering $)$ and filtered image $(7 \times 7$ filtering $)$.
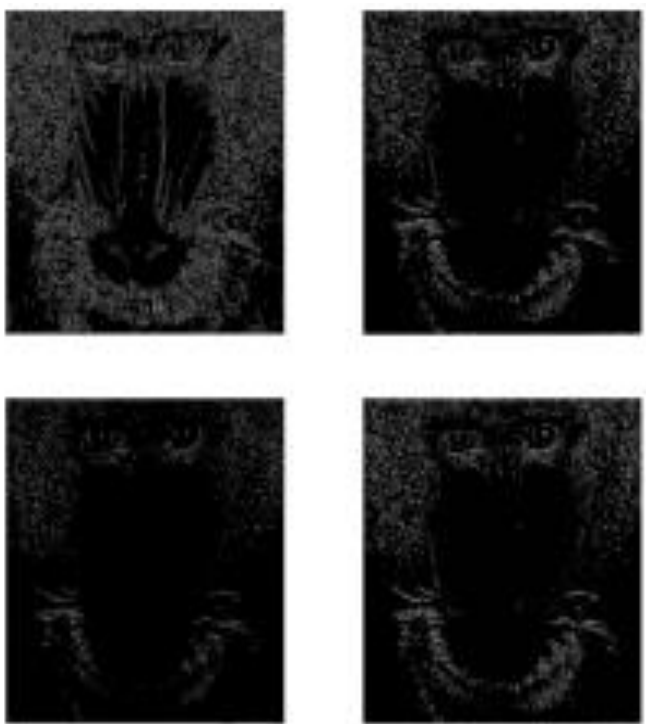

Fig. 8. Results of edge detection with Baboon image. From left-to right-hand side and from top to bottom: result of Laplacian mask, result of Prewitt mask, result of Roberts mask and result of Sobel mask. 
During teaching edge detection with Matlab, we expect that; students should be able to understand how to create edge images using basic filtering methods, as well as observe how the Laplacian operator has the capacity for detecting edges in all directions equally well.
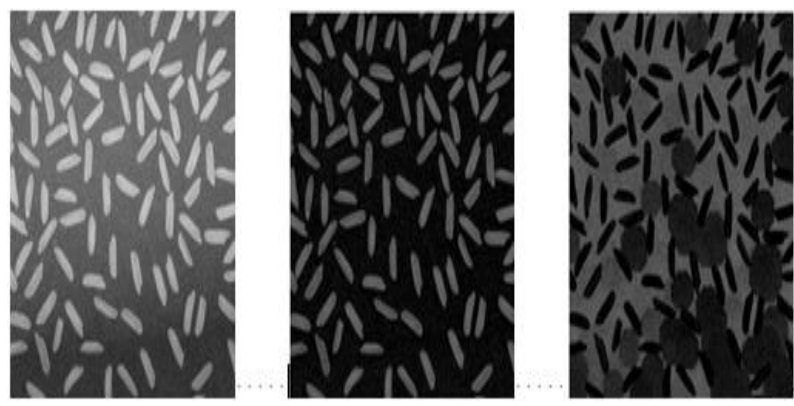

Fig. 9. From left-to right-hand side: original image, top-hat image and bottom-hat image.

TABLE I: PSNRS RESULTS FOR DIFFERENT IMAGES CORRUPTED BY DIFFERENT NOISES

\begin{tabular}{|l|l|l|l|}
\hline Noise & Salt \& pepper & Poisson & Speckle \\
\hline PSNR & 27.0350 & 30.0815 & 24.8662 \\
\hline
\end{tabular}

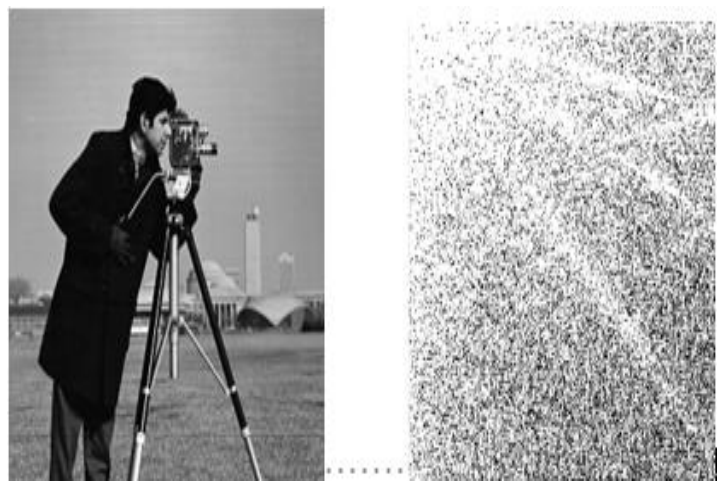

Fig. 10. From left-to right-hand side: Original image and its DCT.
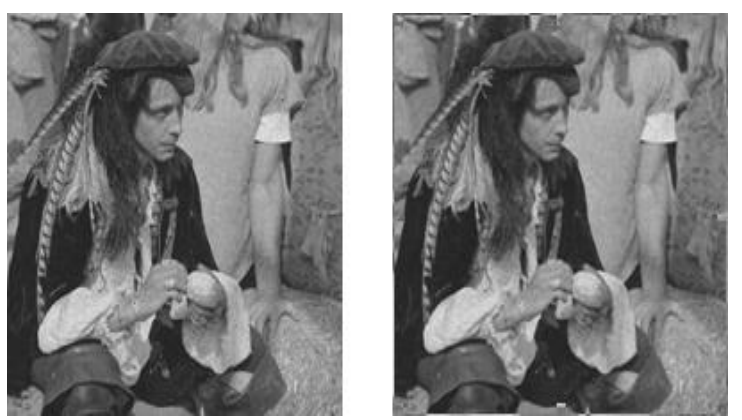

Fig. 11. From left-to right-hand side: Noisy image (Poisson noise) and filtered image.
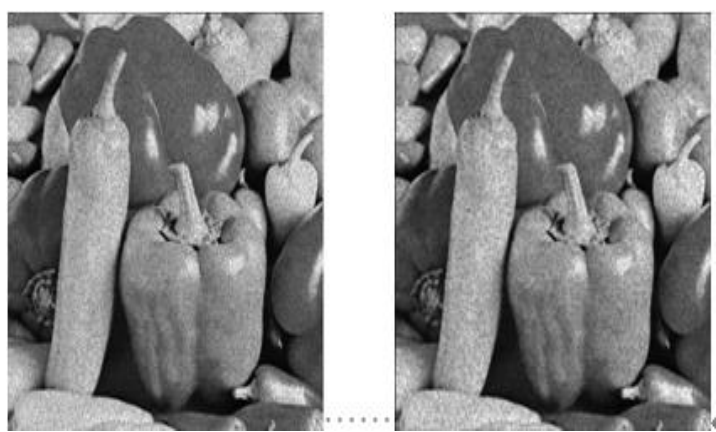

Fig. 12. From left-to right-hand side: Noisy image (Speckle noise (variance $\left.\sigma^{2}=0.04\right)$ ) and filtered image.
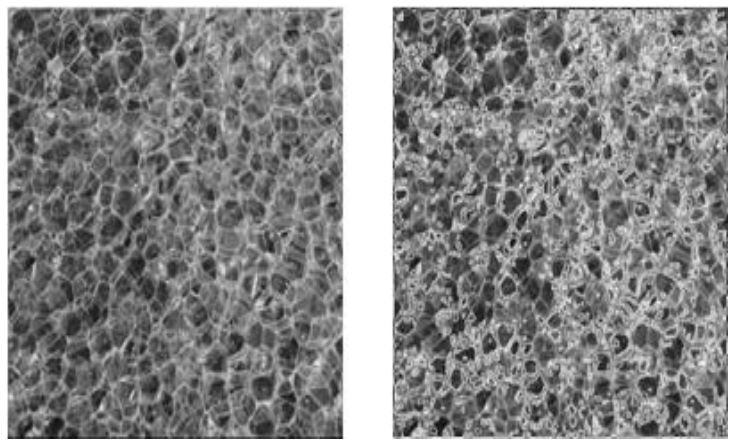

Fig. 13. From left-to right-hand side: Original image, result of Watershed filter [15].

\section{CONCLUSION}

This paper drives the new thoughts on teaching digital image processing topics with Matlab. In this paper, we cover the most important topic in a regular digital image processing curriculum. Proposed approach provides students with a high quality of understanding of the digital image processing topics, in addition to improving their abilities in order to enhance analytical thinking skills. This in turn leads to enhanced creative thinking skills for students and enriched them with more effective programming expertise. We believe that proposed method will helps teachers to improve memory, understanding skills and grasping power in students. As well as harness their creativity and skills to develop the existing digital image processing's algorithms and codes. Experiments based on Matlab are performed on some digital image processing topics such as morphological operations, image denoising, histogram equalization and edge detection. Utilizing Maltlab in teaching digital image processing topics allows students to grasp and master the skills of these topics. By comparing with other existing methods, it is shown that our proposed method "teaching digital Image processing topics via Matlab techniques" has a high ability to promote the undergraduates' theoretical bases, in addition to enhancing their innovative abilities and practical capabilities. Paper gives deep thoughts on teaching digital image processing techniques. We are confident that the ideas in this paper can contribute to the development of current curriculum settlement, in addition to meeting the needs of students from several aspects.

\section{CONFLICT OF INTEREST}

The author declares no conflict.

\section{AUTHOR CONTRIBUTIONS}

There are no co-authors for this paper, so there are no co-authors' contributions.

\section{ACKNOWLEDGMENT}

This work is supported by ANHUI Province Key Laboratory of Affective Computing \& Advanced Intelligent Machine, Grant (No.ACAIM180201).

\section{REFERENCES}

[1] Z. Wang and J. Guo, "Teaching and practice mode reform in digital image processing curriculum," International Journal of Information and Education Technology, 2017, vol. 7, no. 7, pp. 557-560. 
[2] P. A. Larbi, "Advancing microsoft excel's potential for teaching digital image processing and analysis," American Society of Agricultural and Biological Engineers, 2018, vol. 34, no. 2, pp. 263-276.

[3] L. Francisco and C. Campos, "Learning digital image processing concepts with simple scilab graphical user interfaces," European Congress on Computational Methods in Applied Sciences and Engineering, 2018, pp. 548-559.

[4] R. Greenberg, R. A. Kolvoord, M. Magisos, R. G. Strom, and S. Croft, Image processing for teaching, Journal of Science Education and Technology, 1993, pp 469-480.

[5] J. H. Lee, Y. T. Cho, H. Heo, and O. S. Chae, MTES: Visual Programming Environment for Teaching and Research in Image Processing, Springer Berlin Heidelberg, 2005, pp. 1035-1042.

[6] L. Xiaobo and C. Zhao, "Discussion on teaching reform of digital image processing course," Indian Journal of Applied Research, vol. 7, no. 5, 2017, pp. 634-635

[7] Y, Ning, "Exploration of practical teaching reform on signal processing curriculum group," in Proc. the 2015 Conference on Education and Teaching in Colleges and Universities, 2015, pp. 182-184.

[8] Y. Shi, X. Liu, H. Ding, and Y. Xia, "The teaching reform for digital image processing as the discipline's basic course," in Proc. 3rd International Conference on Social Science and Higher Education, 2017, vol. 99, pp. 313-316.

[9] E. J. Leavline and D. A. A. G. Singh, "On teaching digital image processing with MATLAB," American Journal of Signal Processing, 2014, vol. 4, no. 1, pp. 7-15.

[10] H. J. Zhu, J. Zhang, X. R. Zhao, and H. H. Fan, "Teaching and practice of digital image processing curriculum based on engineering application," in Proc. International Conference on Education, Management, Commerce and Society, 2015, pp. 493-497.

[11] C. S. Zuria, J. M. Ramirez, D. Baez-Lopez, and G. E. Flores-Verdad, "MATLAB based image processing lab experiments," in Proc. the 28th Annual Frontiers in Education, 1998, vol. 03, pp. 1255-1258.

[12] R. C. Gonzalez, R. E. Woods, and S. L. Eddins, Digital Image Processing Using MATLAB, Gatesmark Publishing, 2009.

[13] R. C. Gonzalez and R. E. Woods, Digital Image Processing, Publishing House of Electronics Industry, Beijing, China, $3^{\text {rd }}$ edition, 2010.

[14] A. M. Andrew, Introduction to Digital Image Processing with MATLAB, Thomson Course Technology, 2004.

[15] A. A. Yahya, J. Tan, and M. Hu, "A novel model of image segmentation based on watershed algorithm," Advances in Multimedia, 2013, vol. 2013, pp. 1-8.

Copyright (C) 2019 by the authors. This is an open access article distributed under the Creative Commons Attribution License which permits unrestricted use, distribution, and reproduction in any medium, provided the original work is properly cited (CC BY 4.0).

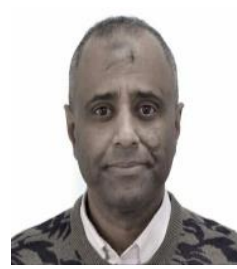

Ali Abdullah Yahya received his Ms. degree in partial differential equation from University of Science and Technology of China, China, in 2010, the $\mathrm{Ph} . \mathrm{D}$ degree in video/image denoising from Hefei University of Technology, China, in 2014, and he worked in Hefei University of Technology as a postdoctoral researcher for two years. Currently he is an assistant professor in Anqing Normal University. 